\section{Linfoma difuso de células grandes B y la importancia de la terapia de soporte en pacientes oncológicos}

\section{Diffuse Large B Cell Lymphoma and the importance of supportive therapy in pediatric oncology.}

Galván-Díaz CA ${ }^{1}$, Cárdenas-Cardós R², Rojas-Maruri $\mathrm{CM}^{3}$, Palma-Rosillo RM ${ }^{4}$, Aguilar-Ortiz MR

\section{PRESENTACIÓN DEL CASO}

Niño de 7 años 6 meses de edad, con historia de cuatro meses de evolución caracterizada por constipación nasal, epistaxis y rinorrea amarillenta bilateral; fiebre no cuantificada de predominio nocturno y pérdida de peso no cuantificada. Estos síntomas se presentaban al menos una vez por semana de manera continua. Examen físico: aumento de volumen de la región malar derecha y el párpado inferior ipsi-lateral, con dolor a la palpación; adenomegalias bilaterales cervicales y sub-mandibulares con diámetro de 0.5 a $1 \mathrm{~cm}$ de consistencia blanda. Una radiografía posteroanterior de tórax no mostró masa mediastinal. La biometría hemática reportó $12.9 \mathrm{~g} / \mathrm{dL}$ de hemoglobina, 8,600/ $\mathrm{mm}^{3}$ leucocitos, $6,600 / \mathrm{mm}^{3}$ neutrófilos, $1,900 / \mathrm{mm}^{3}$ linfocitos, $100 /$ $\mathrm{mm}^{3}$ monocitos y $44,6000 / \mathrm{mm}^{3}$ de plaquetas. La tomografía de emisión de positrones con flúor 18 unido a fluordeoxiglucosa, en combinación con la tomografía simple $\left({ }^{18} \mathrm{~F}\right.$-FDG PET/CT), mostró una lesión lítica que ocupaba el etmoides anterior y posterior, el seno maxilar en forma bilateral y extensión al piso de la órbita; sin afectar el nervio óptico; el tumor ocupa la fosa nasal derecha e izquierda y la nasofaringe con SUVmax de 16.31 denotando un proceso de hiper-metabolismo celular, altamente sugestivo de malignidad-neoplasia.

El diagnóstico se realizó en una biopsia-incisional: se trataba de una tumoración de aspecto friable, por lo que se colocó un
${ }^{1}$ Residente de cuarto año de subespecialidad en Oncología Pediátrica.

2Jefe de Servicio, Oncología Pediátrica, Subdirección de Hemato-Oncología.

${ }^{3}$ Médico adscrito, Departamento de Anatomía Patológica.

${ }^{4}$ Jefa de Servicio, Servicio de Medicina Nuclear.

${ }^{5}$ Médico adscrito al servicio de Oncología Pediátrica, Subdirección de Hemato-Oncología.

Instituto Nacional de Pediatría.

Recibido: 12 de mayo del 2016

Aceptado: 25 de mayo del 2016

Correspondencia

Dr. Marco Rodrigo Aguilar Ortiz

Médico adscrito al servicio de Oncología Pediátrica Instituto Nacional de Pediatría

teléfono 10840900 ext. 1339

marconco.inp@gmail.com

Este artículo debe citarse como

Galván-Díaz CA, Cárdenas-Cardós R, Rojas-Maruri $\mathrm{CM}$, Palma-Rosillo RM, Aguilar-Ortiz MR. Linfoma difuso de células grandes $\mathrm{B}$ y la importancia de la terapia de soporte en pacientes oncológicos. Acta Pediatr Mex. 2016;37(4):222-227. 
taponamiento anterior del lado derecho. Tres días después del evento quirúrgico, aún sin informe histopatológico, el paciente mostró incremento de la sintomatología obstructiva debido a inflamación y al crecimiento del tumor. Por tratarse de una urgencia obstructiva de la vía aérea superior se inició citorreducción con quimioterapia: ciclofosfamida $200 \mathrm{mg} / \mathrm{m}^{2} /$ día por dos días y dexametasona $10 \mathrm{mg} / \mathrm{m}^{2} /$ día durante 5 días. La respuesta clínica inicial fue adecuada.

El informe histopatológico fue linfoma no Hodgkin difuso de células grandes B; por lo que se inició tratamiento con protocolo técnico para linfoma no Hodgkin B; se administró Curso A: ciclofosfamida, doxorrubicina, vincristina y terapia intratecal con metotrexate/citarabina, sin complicaciones.

Como parte del manejo inicial, durante la fase de citorreducción y el inicio de la quimioterapia, el tratamiento fue hiper-hidratación e inhibidor de xantina-oxidasa, además de vigilancia estrecha de los parámetros de síndrome de lisis tumoral con determinaciones frecuentes de ácido úrico, fósforo y potasio (Cuadro 1), debido al alto riesgo de presentar síndrome de lisis tumoral por la biología misma de esta neoplasia.

El paciente tuvo buena evolución de manera inicial, gracias al tratamiento y la prevención oportuna a las urgencias oncológicas que acompañan a esta patología. Al tercer día de iniciada la quimioterapia (Curso A) el paciente tuvo fie-

Cuadro 1. Determinación de parámetros de lisis tumoral durante el inicio del tratamiento

\begin{tabular}{lllll}
\hline Basal & 4.8 & 9.6 & 5.1 & 4 \\
\hline Máximos al inicio de la CR & 4.7 & 9.2 & 4.7 & 2.9 \\
\hline Máximos al fin de la CR & 2.9 & 8.2 & 2.2 & 2.2 \\
\hline Máximos semana 1 de QT & 3.2 & 7.4 & 2.3 & 1.5
\end{tabular}

CR: citorreducción; QT: quimioterapia Curso A. bre: $38.8^{\circ} \mathrm{C}$, sin evidencia de un foco infeccioso. Se hicieron estudios de laboratorio y cultivos: hemoglobina $12.6 \mathrm{~g} / \mathrm{dL}$, leucocitos en 4,600/ $\mathrm{mm}^{3}$, neutrófilos $2,500 / \mathrm{mm}^{3}$, linfocitos 1,900 / $\mathrm{mm}^{3}$, monocitos $140 / \mathrm{mm}^{3}$ y plaquetas $31,6000 /$ $\mathrm{mm}^{3}$, examen general de orina sin alteraciones y proteína $C$ reactiva en $3 \mathrm{mg} / \mathrm{dL}$. Se inició la administración de antibióticos: cefuroxima y clindamicina por sospecha de proceso infeccioso en vías respiratorias superiores, debido al taponamiento nasal temporal. La evolución posterior fue tórpida: fiebre elevada y mucositis oral grado IV. El hemocultivo inicial reveló Enterobacter aerogenes; el urocultivo mostró Pseudomonas aeruginosa. Tuvo mala evolución del proceso infeccioso y agravamiento de las lesiones en cavidad oral que requirieron aporte nutricional por vía parenteral. Debido a la evolución clínica se agregaron al esquema antibióticos: vancomicina y meropenem, además de cobertura antifúngica con fluconazol; sin embargo, los datos de sepsis progresaron hasta la falla hemodinámica, por lo que fue tratado en la unidad de terapia intensiva. Posteriormente presentó choque séptico y falleció.

\section{Comentario radiológico}

\section{Ver Figuras 1 a 3.}

\section{Hallazgos anatomopatológicos}

Autopsia del paciente de edad aparente igual a la cronológica. Existían úlceras en labios y lengua, con lesiones ovoides de color blanquecino en esta última (Figura 4). El colon tenía la mucosa material de aspecto membranoso de coloración blanco-amarilla (Figura 5). El hígado era de coloración amarillo claro, con disminución importante de la consistencia.

Microscopía. Médula ósea con hipoplasia moderada, así como depleción linfocítica en ganglios. El epitelio esofágico y de la lengua 


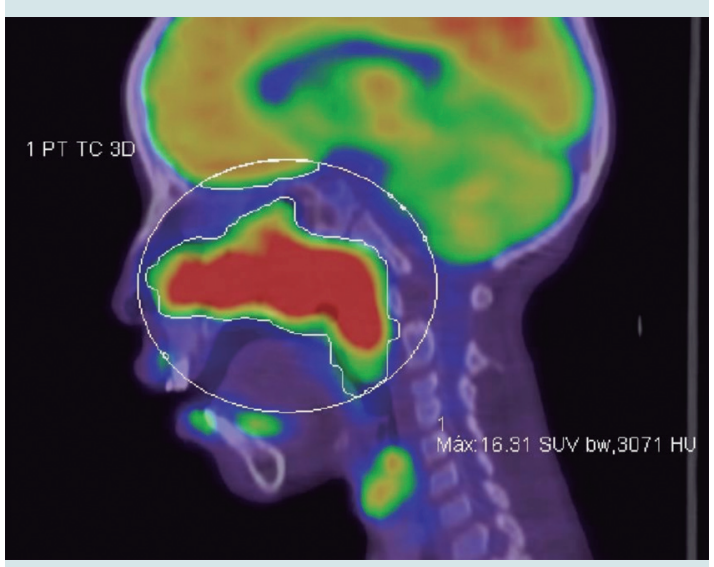

Figura 1. Tomografía de emisión de positrones con flúor 18, unido a fluordeoxiglucosa, en combinación con tomografía simple; proyección sagital: tumor en región derecha de la cara que involucra tejidos blandos y hueso desde la cavidad nasal derecha hasta arriba de la raíz de premolar y canino superiores derechos y lateralmente hacia hueso cigomático; con hipermetabolismo importante (SUVmax de 16.3).

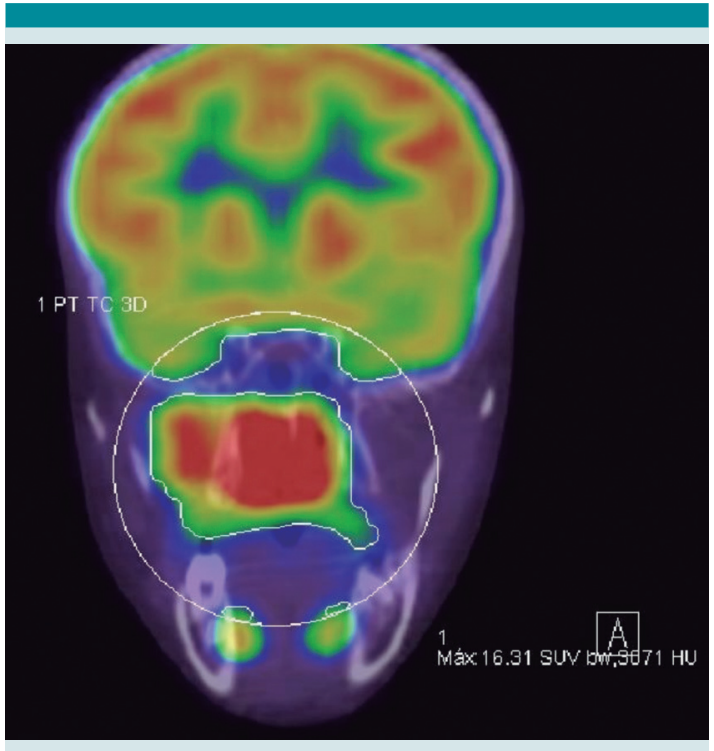

Figura 2. Tomografía de emisión de positrones con flúor 18, unido a fluordeoxiglucosa, en combinación con tomografía simple; corte coronal: se aprecia la extensión de la lesión hasta el piso de la orbita ipsilateral, región malar y maxilar; esta proyección también con SUVmax de 16.3.

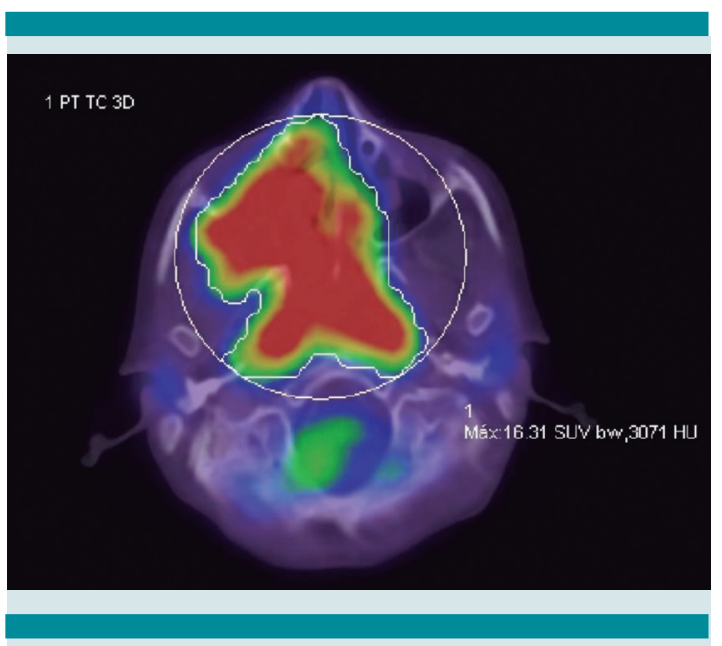

Figura 3. Tomografía de emisión de positrones con flúor 18, unido a fluordeoxiglucosa, en combinación con tomografía simple; plano axial: extensión anteroposterior del tumor limitada a regiones mencionadas en las figuras anteriores, SUVmax de 16.3.

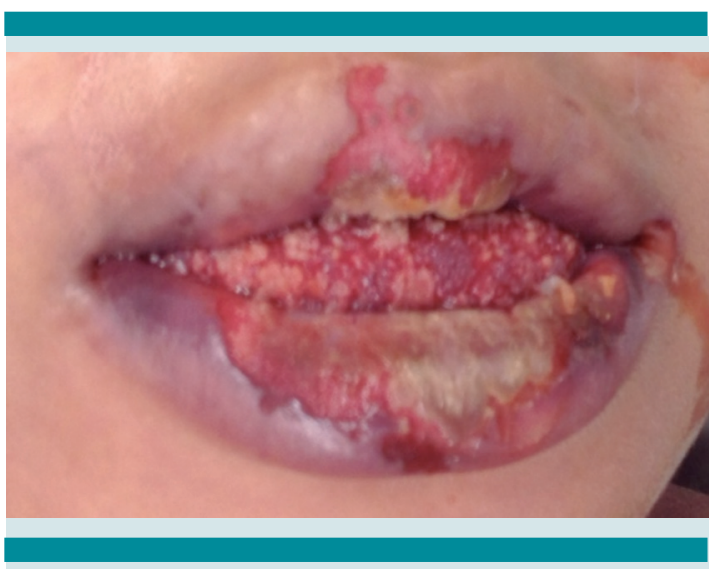

Figura 4. Úlceras en labios y lengua (esta última con lesiones nodulares de color blanquecino)

mostraron inclusiones virales de citomegalovirus y de herpes virus (Figura 6). El colon mostraba necrosis focal de la mucosa, con presencia de membranas adherentes y colonias bacterianas Gram negativas (Figura 7).

El tejido hepático mostró necrosis extensa de hepatocitos, sin evidenciarse datos de infección viral o microorganismos debido a lo dañado del tejido. 
Galván-Díaz CA et al. LDCGB y terapia de soporte en oncología pediátrica

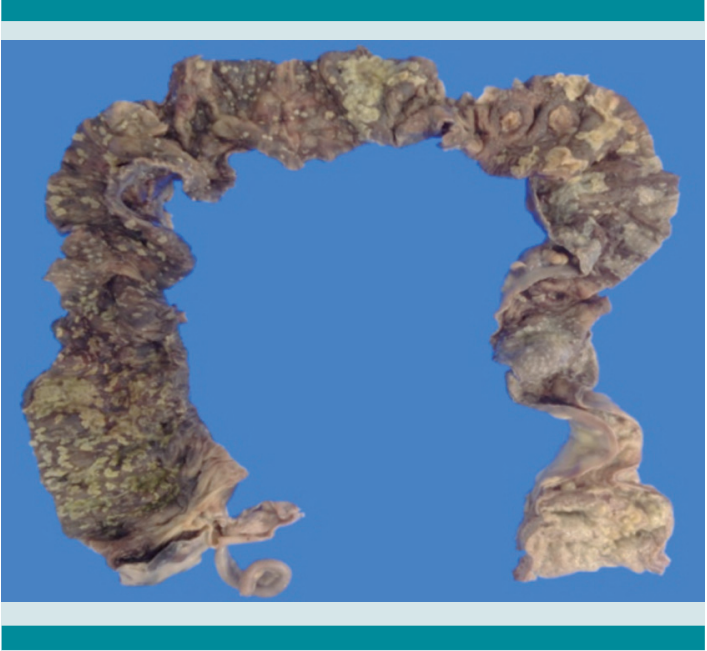

Figura 5. Colon con membranas blanco-amarillentas en la superficie mucosa.

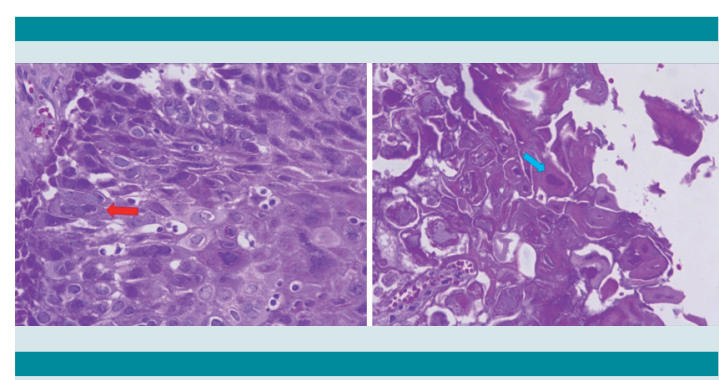

Figura 6. Células epiteliales multinucleadas en aspecto de "bolsa de canicas" características de infección por virus Herpes (izquierda). Inclusión citomegálica indicativa de infección por citomegalovirus (derecha).

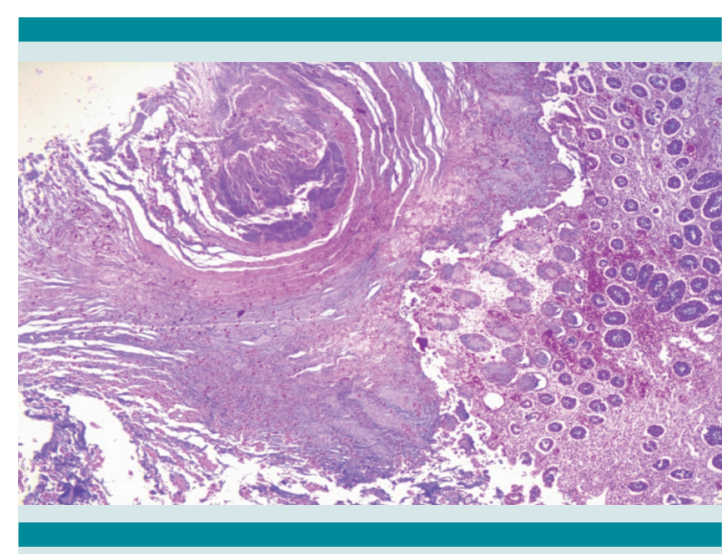

Figura 7. Mucosa de colon con necrosis focal y membranas en su superficie con bacterias Gram negativas.
Los hallazgos: hipoplasia de médula ósea y depleción linfocítica son complicaciones secundarias al tratamiento del proceso linfoproliferativo del paciente. Dichas complicaciones favorecieron la infección por citomegalovirus y herpes virus en boca y esófago, así como la infección bacteriana en colon, lo que llevó al paciente a la muerte. La necrosis hepática sub masiva pudo haber sido ocasionada por un agente viral de los arriba mencionados; sin embargo, debido al daño hepático observado no se pudo demostrar el mismo. El resto de los hallazgos se enlistan en el Cuadro 2.

\section{DISCUSIÓN}

En la mayoría de las ocasiones las urgencias oncológicas acompañan a los pacientes recientemente diagnosticados con cáncer, por lo que el equipo involucrado en la atención de éstos debe estar capacitado para la prevención, de-

Cuadro 2. Diagnósticos anatomopatológicos finales

I. Linfoma no Hodgkin difuso de células grandes B extranodal, primario de región nasofaríngea (B14-1983), tratado con quimioterapia (ciclofosfamida, doxorrubicina, vincristina y TIT con metotrexate/citarabina)

-Médula ósea con hipoplasia moderada -Depleción linfocítica en ganglios linfáticos - Historia de respuesta clínica a quimioterapia -Infección por citomegalovirus y herpes virus en lengua y esófago

-Pancolitis neutropénica con pseudomembranas (historia clínica de neutropenia)

II. Necrosis hepática submasiva no determinada

III. Datos anatómicos de choque:

-Necrosis tubular aguda

-Miopatía hipóxico isquémica extensa

-Necrosis superficial de mucosa de intestino delgado

IV. Gastritis crónica ulcerada leve focal

V. Cistitis crónica leve ulcerada leve focal

VI. Laringitis leve ulcerada

VII. Tráqueobronquitis leve ulcerada

VIII. Hemorragia subpleural leve focal

IX. Cardiomegalia leve a expensas de dilatación de cavidades derechas

X. Hemorragia parenquimatosa testicular bilateral leve 
tección y manejo oportuno de las mismas en su práctica diaria. ${ }^{1}$

El caso presentado es un ejemplo de la importancia del diagnóstico y la atención oportuna de las mismas:

1. Obstrucción de la vena cava y presencia de masa mediastinal, ${ }^{2}$ la cual fue descartada por clínica y radiografía de tórax ${ }^{3}$ a su diagnóstico.

2. Obstrucción aguda de la vía aérea ${ }^{4}$ ocasionada por el tumor, lo obligó al inicio temprano de la citorreducción con quimioterapia.

3. La obstrucción intestinal y la compresión medular o ambas fueron complicaciones no presentes en el paciente por la localización del tumor.

4. El síndrome de lisis tumoral ${ }^{5}$ es la urgencia oncológica que ocurre con mayor frecuencia en pacientes con linfoma no Hodgkin debido a la biología tumoral de esta neoplasia. Por tal motivo, es indispensable que el pediatra esté familiarizado con su diagnóstico y tratamiento, previniéndola en todos los pacientes mediante el manejo con hiper-hidratación cuando la función renal y estado hemodinámico lo permitan; en nuestro caso estuvo ausente quizá por las medidas instauradas oportunamente. ${ }^{6}$

5. La mucositis también es uno de los efectos secundarios más comunes y molestos en la terapéutica del cáncer, ${ }^{7}$ pudiendo ser tan dolorosa que comprometa la nutrición y la higiene oral, incrementando así el riesgo de infección local y sistémica, ${ }^{8}$ por lo que también deberá considerarse importante en el tratamiento de soporte que debe incluir medidas locales, terapia nutricional y control del dolor.
En este caso, las complicaciones infecciosas causaron la muerte del paciente. La fiebre en los pacientes oncológicos es un dato de alarma, ya que por lo general precede a un proceso infeccioso que puede poner en riesgo la vida, ${ }^{9} \mathrm{y}$ a menudo es el único signo de infección potencialmente fatal (la tasa de sepsis en niños entre 1 a 9 años es de $12.8 \%$ y entre 10 a 19 años es de $17.4 \%)^{10}$

Se identifican tres momentos de alto riesgo para la aparición de fiebre:

1. Al inicio del tratamiento por el compromiso inmunológico que el cáncer descontrolado genera.

2. Debido al tratamiento, por la neutropenia causada por la quimioterapia. ${ }^{11}$

3. En algunas neoplasias las mismas células tumorales son capaces de liberar citocinas y generar respuesta inflamatoria sistémica. ${ }^{12}$

La instauración temprana de antibióticos de amplio espectro puede ir en contra de los cánones de brindar la terapia antibiótica más específica y de menor espectro posible para evitar resistencia a los antibióticos; sin embargo, en pacientes en fases iníciales de tratamiento para el cáncer ${ }^{13}$ se debe considerar una terapia dirigida de acuerdo con el riesgo de aplasia medular, así como individualizar la selección de antibióticos de acuerdo con cada hospital y con la epidemiología del mismo.

Una peculiaridad importante de este caso es la evolución de la mucositis; cuando esta complicación es de larga evolución (de 8 hasta 12 días vs. 1 a 2.5 días del promedio en la mayoría de los casos) o no presenta buena respuesta al tratamiento antibiótico se ha relacionado con infección por Herpes Simple Virus corroborado con hisopado buco-faríngeo a pesar de serolo- 
gía negativa para este virus. ${ }^{14}$ Se han descrito distintas estrategias preventivas como detección y manejo oportuno de gingivitis o infecciones orales, crioterapia, aseos bucales protocolizados, suplementos sistémicos de zinc y factor de crecimiento de queratinocitos (palifermin), cuyas recomendaciones deben ser individualizadas para cada paciente, así como tratamiento oportuno (indicado desde que se presenta mucositis grado II) con aseos bucales (tratamiento tópico) con morfina por su efecto analgésico potente y doxepina como antihistamínico, y analgésicos sistémicos. ${ }^{15}$

Estas medidas junto con la vigilancia en unidades de terapia intensiva al iniciar el tratamiento antineoplásico son medidas que se deben instaurar de manera temprana. El caso presentado nos obliga a analizar las estrategias de manejo y la importancia de la individualización y especial monitorización en pacientes con mala evolución o con respuesta atípica a la terapéutica empleada. Las complicaciones presentadas en el paciente del caso son frecuentes durante el tratamiento antineoplásico; sin embargo, la evolución no fue la esperada. Se deben plantear estrategias para detectar pacientes de alto riesgo a desarrollar complicaciones como mucositis exacerbada por infecciones virales e insistir en cobertura antibiótica de amplio espectro inicial. Hoy en día los avances en las estrategias terapéuticas contra el cáncer permiten una alta supervivencia que no debemos permitir se vea mermada por limitar su manejo de soporte.

\section{REFERENCIAS}

1. Fisher MJ, Rheingold SR. Oncologic Emergencies. In: Principles and Practice of Pediatric Oncology, 6th, Pizzo PA, Poplack DG (Eds), Lippincott Williams and Wilkins, Philadelphia 2011. p.1125.

2. Walji N, Chan AK, Peake DR. Common acute oncological emergencies: diagnosis, investigation and management. Postgrad Med J. 2008;84:418-427.
3. Wilson LD, Detterbeck FC, Yahalom J. Superior vena cava syndrome with malignant causes. N Engl JMed. 2007;356:1862-1869.

4. Ben-Ari J, Schonfeld T, Harlev E, Steinberg R, Yaniv I, Katz J, Schwartz M, Freud E. Life threatenin gairway obstruction secondary to mass in children - a preventable event? Pediatric Emergency Care. 2005;21(7):427-430.

5. Cairo, M.S., Coiffier, B., Reiter, A. and Younes, A. Recommendations for the evaluation ofrisk and prophylaxis of tumor lysis syndrome (TLS) in adults and children with malignant disease: an expert TLS panel consensus. British Journal of Hematology. 2010;149; 578-586.

6. Coiffier B, Altman A, Pui C-H.and Cairo MS. Guidelines for the management of pediatric and adult tumor lysis syndrome: an evidence-based review. Journal of Clinical Oncology. 2008;26(16):2767-2778.

7. Sonis ST. A biological approach to mucositis. J Support Oncol. 2004; 2:21-32.

8. Lalla RV, Sonis ST, Peterson DE Management of oral mucositis in patients who have cancer. Dent Clin North Am. 2008:52;61-77.

9. Castagnola E, Fontana V, Caviglia I, et al. A prospective study on the epidemiology of febrile episodes during chemotherapy-induced neutropenia in children with cancer or after hemopoietic stem cell transplantation. Clin Infect Dis. 2007;45:1296.

10. Mendes A, Sapolnik R, Mendonca N. New guidelines for the clinical management of febrile neutropenia and sepsis in pediatric oncology patients. Jornal de Pediatria. 2007;83(2):S54.

11. Lehrnbecher T, Foster $\mathrm{C}$, Vazquez $\mathrm{N}$ et al. Therapy induced alterations in host defense in children receiving therapy for cancer. J Pediatr Hematol Onco. 1997;119:399-417.

12. Phillips RS, Wade R, Lehrnbecher $\mathrm{T}$ et al. Systematic review and meta-analysis of the value of initial biomarkers in predicting adverse outcome in febrile neutropenic episodes in children and young people with cancer. BMC Med. 2012;10:6.

13. Hughes W, Armstrong D, Bodey G, Bow E, Brown A, et al. Guide lines for the Use of Antimicrobial Agents in Neutropenic Patients with Cancer. CID. 2002;34:730-751.

14. Ramphal R, Grant RM, Dzolganovski B, et al. Herpes simplex virus in febrile neutropenic children undergoing chemotherapy for cancer: a prospective cohort study. Pediatr Infect Dis J. 2007;26:700.

15. Lalla RV, Bowen J, Barasch A, et al. MASCC/ISOO clinical practice guide lines for the management of mucositis secondary to cancer therapy. Cancer. 2014;120:1453. 